\title{
CONTOUR INTEGRATION IN THE THEORY OF THE SPHERICAL PENDULUM AND THE HEAVY SYMMETRICAL TOP
}

\author{
BY \\ WALTER KOHN
}

INDEX

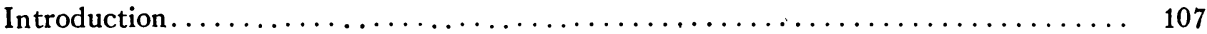

1. History of the method and contents of the present paper. Tables of results.... 107

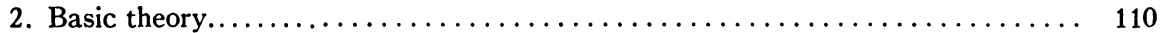

Part I. The spherical pendulum $\ldots \ldots \ldots \ldots \ldots \ldots \ldots \ldots \ldots \ldots \ldots \ldots \ldots \ldots \ldots \ldots \ldots \ldots$

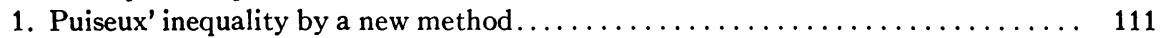

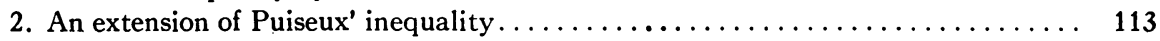

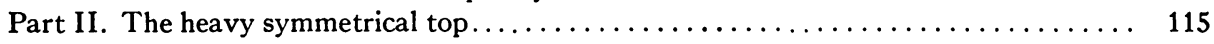

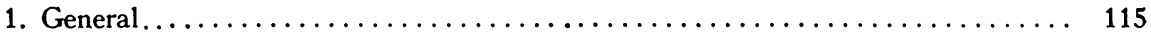

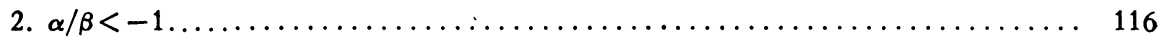

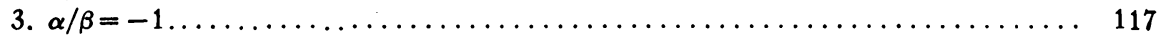

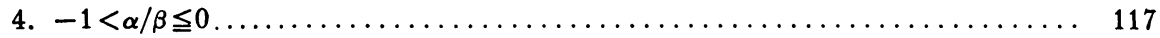

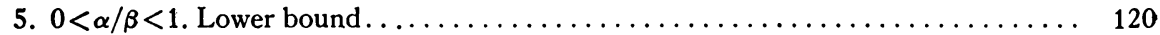

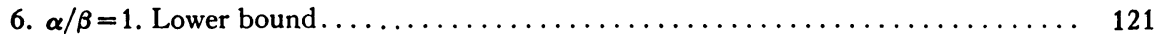

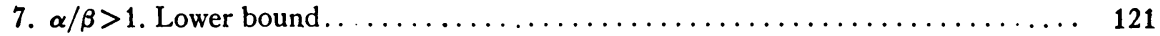

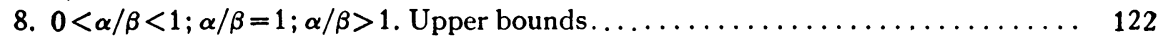

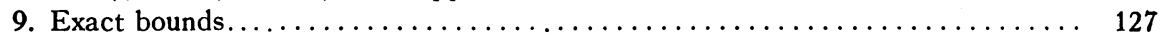

10. Continuity of $\Phi$ for a fixed $\alpha / \beta \ldots \ldots \ldots \ldots \ldots \ldots \ldots \ldots \ldots \ldots \ldots \ldots \ldots \ldots \ldots$

Appendix: The retrogradely precessing heavy symmetrical top and the spherical pen-

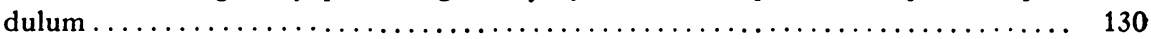

\section{INTRODUCTION}

1. History of the method and contents of the present paper. Tables of results. At the close of the last century J. Hadamard $\left({ }^{1}\right)$ conceived the ingenious idea of applying contour-integration to the theory of tops. This approach enabled him to give an elegant proof of a result which had previously been established by Halphen $\left({ }^{2}\right)$, namely that the total advance in azimuth of a heavy symmetrical top describing loops has the same sense as its precession on the lowest level. Hadamard did not pursue the possibilities of his method any further but soon St. Germain $\left({ }^{3}\right)$ took up his idea and adapted it to the motion of a spherical pendulum. He was immediately led to Halphen's $\left({ }^{4}\right)$ well known result, $\Phi<\pi$, in the form

Presented to the Society, November 25, 1944; received by the editors January 26, 1945.

(1) J. Hadamard, Bull. Sci. Math. (2) vol. 19 (1895) p. 228.

(2) Halphen, Traité des fonctions elliptiques, vol. 2, 1888, p. 113.

(3) A. de St. Germain, Bull. Sci. Math. (2) vol. 20 (1896) p. 114 and vol. 22 (1898) p. 95.

(4) Halphen, Traité des fonctions elliptiques, vol. 2, 1888, p. 128. 


$$
\Phi=\pi-I,
$$

where $\Phi$ is the advance in azimuth corresponding to the passage of the pendulum from the lowest to the highest level of its motion, and $I$ is a positive integral. Later he showed by a somewhat laborious computation that $I$ was smaller than $\pi / 2$ and thus arrived at the inequality $\Phi>\pi / 2$, originally due to Puiseux $\left(^{5}\right)$. In recent years interest in this method has been revived by a publication of A. Weinstein $\left(^{6}\right)$ in which he gave a simpler proof of Puiseux' inequality by introducing, as part of the contour, a vertical straight line instead of the infinite horizontal cut used by Hadamard and St. Germain.

TABLE I

THE SPHERICAL PENDULUM

\begin{tabular}{|c|c|c|c|}
\hline$L$ & $U$ & $z_{1}+z_{2}$ & $\Phi_{0}$ \\
\hline$\pi / 2(5)$ & $\pi(4)$ & $<0\left({ }^{7}\right)$ & $\pi / 2<\Phi_{0} \leqq \Phi<\pi$ \\
\hline
\end{tabular}

TABLE II

THE HEAVY SYMMETRICAL TOP

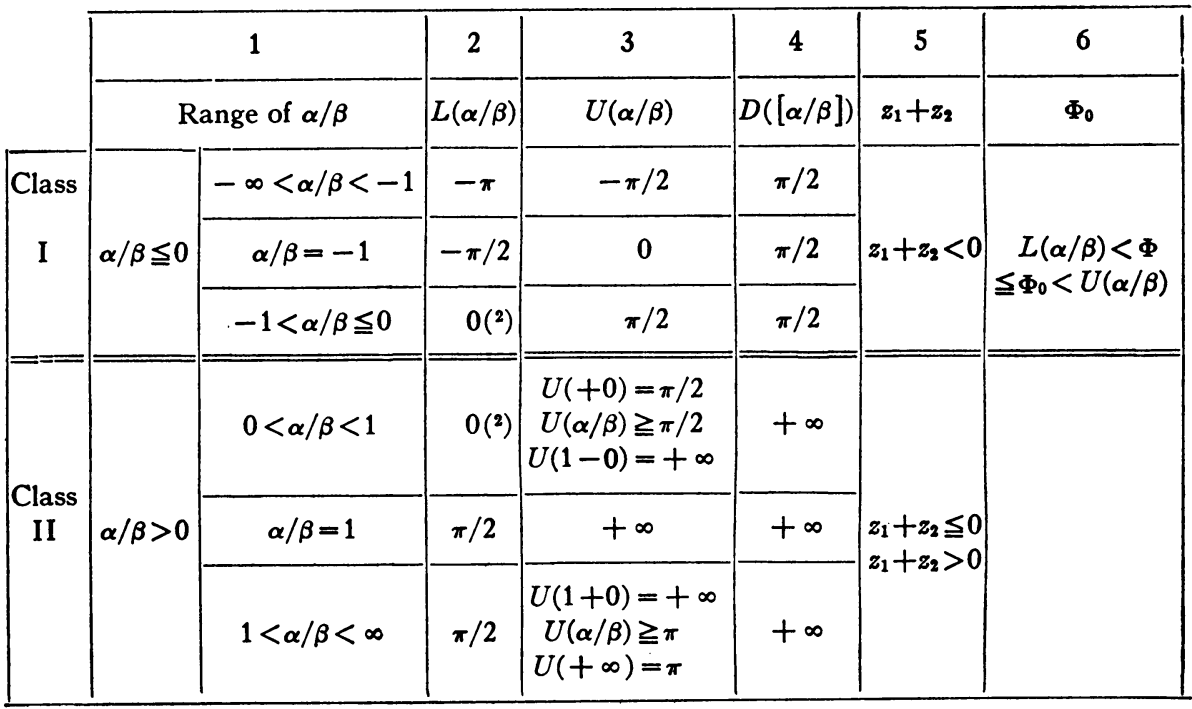

In the present communication the method of complex integration as applied to tops and the spherical pendulum is further developed. Part I deals with Puiseux' inequality for the spherical pendulum. First an alternative proof, based on a simple decomposition and less elaborate than any of those mentioned above, is given. In the second section a slight modification of

(5) Puiseux, Journal de Liouville vol. 7 (1842) p. 517.

(ङ) A. Weinstein, Amer. Math. Monthly vol. 49 (1942) p. 521. 
Weinstein's contour is introduced and with its aid a new result, which is stronger than Puiseux' inequality, is established.

Part II contains a systemmatic examination of the bounds of the angle $\Phi$ in the case of the heavy symmetrical top. By the use of Hadamard's horizontal cuts, Weinstein's vertical line as well as our method of decomposition, limits of $\Phi$ are obtained, which are shown to be actually the greatest lower and least upper bounds respectively.

A short appendix deals with the similarities of the motions of a retrogradely precessing top and the spherical pendulum.

The results obtained in the present paper as well as the known results (marked by references) pertaining to the subject, are listed in Tables I and II. Diag. 1 shows values of $\Phi$ in the different types of motion of a heavy symmetrical top.

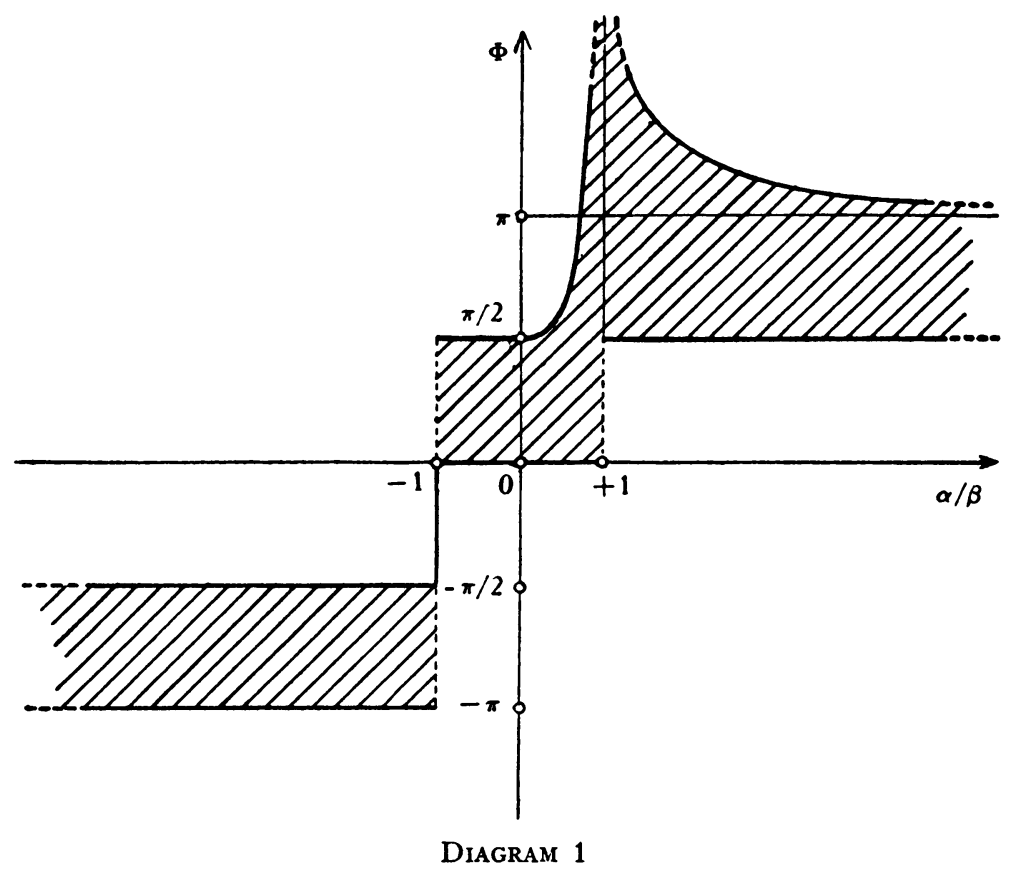

Definition of symbols: $L$ and $U$ are the greatest lower and least upper bound of the advance in azimuth of a spherical pendulum; $z_{1}$ and $z_{2}$ are the lowest and highest levels of motion respectively; $\Phi_{0}$ and $\Phi$ are the advances in azimuth from $z_{1}$ to 0 , and from $z_{1}$ to $z_{2}$ respectively; $\alpha$ and $\beta$ are the constant components of the angular velocity about the vertical axis and axis of symmetry respectively ( $\beta$ is always assumed not less than 0$) L(\alpha / \beta)$ and $U(\alpha / \beta)$ are the greatest lower and least upper bounds of $\Phi$ for all motions of a heavy 
symmetrical top with a given $\alpha / \beta ; D([\alpha / \beta])$ is the maximum difference, $U(\alpha / \beta)-L(\alpha / \beta)$, for $\alpha / \beta$ on the interval indicated (Column 1, Table II).

Table II shows that the motions of the heavy symmetrical top fall into two classes according as $\alpha / \beta \leqq 0$ or $\alpha / \beta>0$. In the first class $z_{1}+z_{2}<0$ and $D([\alpha / \beta])=\pi / 2$, while in the second class the sign of $z_{1}+z_{2}$ may be positive or negative and $D([\alpha / \beta])=+\infty$.

In diag. 1 the upper boundary, $U(\alpha / \beta)$, of the shaded area, corresponding to positive values of $\alpha / \beta$, is a single-valued function of $\alpha / \beta$ with the properties given in column 3 , Table II. Its exact nature is not known. For $\alpha / \beta=-1$, $\Phi$ assumes all values between $-\pi / 2$ and 0 ; for $\alpha / \beta=1$, all values greater than $\pi / 2$ (cf. columns 2 and 3 , Table II).

The author wishes to express his sincerest thanks to Professor A. Weinstein, for his interest and encouragement.

2. Basic theory. The following is a summary of the theory of tops and the spherical pendulum as far as we shall require it as a basis for our further work.

The vertex of a heavy symmetrical top moves according to the equations $\left({ }^{7}\right)$ :

$$
\left(\frac{d z}{d t}\right)^{2}=\frac{1}{A}\left(2 E-\frac{\beta^{2}}{C}-2 m g a z\right)\left(1-z^{2}\right)-\frac{1}{A^{2}}(\alpha-\beta z)^{2}=f(z)
$$

and

$$
\frac{d \phi}{d t}=\frac{\alpha-\beta z}{A\left(1-z^{2}\right)}
$$

where $z$ is the cosine of the angle of nutation; $t$ is the time; $A$ and $C$ are the moments of inertia about a transverse axis and the axis of symmetry respectively; $E$ is the total energy of the top; $\alpha$ and $\beta$ are the constant components of the angular momentum about the vertical and the axis of symmetry respectively; $m$ is the mass of the top; $g$ the acceleration due to gravity; $a$ the distance of the mass centre from the point of suspension; and $\phi$ the angle of azimuth.

The three roots of $f(z)=0$ are real and satisfy the inequalities $-1 \leqq z_{1} \leqq z_{2}$ $\leqq+1 \leqq z_{3}$. The roots $z_{1}$ and $z_{2}$ represent the lowest and highest levels of the motion.

Equations (1) and (2) may be combined to give

$$
\frac{d \phi}{d z}=\frac{d \phi}{d t} \frac{d t}{d z}=\frac{\alpha-\beta z}{A\left(1-z^{2}\right)[f(z)]^{1 / 2}}=g(z),
$$

$\left({ }^{7}\right)$ For a simple and complete development of these equations see J. L. Synge and B. A. Griffith, Principles of mechanics, 1942, pp. 418-421, also pp. 369-372. 
where we choose the positive sign for the root of $f(z)$ and thus limit ourselves to the motion from $z_{1}$ to $z_{2}$ during which $d z / d t=[f(z)]^{1 / 2}$ is positive. The total advance in azimuth during the passage from $z_{1}$ to $z_{2}$ is

$$
\Phi=\phi\left(z_{2}\right)-\phi\left(z_{1}\right)=\int_{z 1}^{z_{2}} \frac{\alpha-\beta z}{A\left(1-z^{2}\right)[f(z)]^{1 / 2}} d z=\int_{z 1}^{z 2} g(z) d z .
$$

The corresponding equations for the spherical pendulum may be immediately obtained by setting $\beta=0$. Thus (1), (3) and (4) become

$$
\begin{gathered}
\left(\frac{d z}{d t}\right)^{2}=\frac{1}{A}(2 E-2 m g a z)\left(1-z^{2}\right)-\frac{\alpha^{2}}{A^{2}}=f(z), \\
\frac{d \phi}{d z}=\frac{\alpha}{A\left(1-z^{2}\right)[f(z)]^{1 / 2}}=g(z),
\end{gathered}
$$

and

$$
\Phi=\phi\left(z_{2}\right)-\phi\left(z_{1}\right)=\int_{z 1}^{z_{2}} \frac{\alpha}{A\left(1-z^{2}\right)[f(z)]^{1 / 2}} d z=\int_{z 1}^{z_{2}} g(z) d z .
$$

\section{PART I. The SPherical PENDUlum}

I.1. Puiseux' inequality by a new method. As mentioned in the introduction, Puiseux $\left({ }^{5}\right)$, in 1842 , published the theorem that a spherical pendulum, in passing from the lower to the upper level of its motion, advances in azimuth by an angle greater than $\pi / 2$. From a mathematical point of view Puiseux' inequality means that if we take $\alpha>0$,

$$
\Phi=\int_{z 1}^{z_{2}} g(z) d z=\int_{z 1}^{z_{2}} \frac{\alpha}{A\left(1-z^{2}\right)[f(z)]^{1 / 2}} d z>\pi / 2,
$$

where $f(z)$ is defined by equation (5). This result allows of a simple proof by contour-integration after the factor $1 /\left(1-z^{2}\right)$ in $g(z)$ has been decomposed into partial fractions and thus $g(z)$ itself split into two parts, namely

$$
g(z)=\phi_{1}(z)+g_{2}(z)
$$

where

$$
g_{1}(z)=-\alpha / 2 A(z-1)[f(z)]^{1 / 2}
$$

and

$$
g_{2}(z)=\alpha / 2 A(z+1)[f(z)]^{1 / 2}
$$

Setting

$$
\int_{z 1}^{z 2} g_{k}(z) d z=\Phi_{k}, \quad k=1,2,
$$


we have

$$
\Phi=\Phi_{1}+\Phi_{2} .
$$

We shall now estimate $\Phi_{1}$ and $\Phi_{2}$ separately. $g_{1}(z)$ is positive during the whole motion (see (9)) and consequently, by (11),

$$
\Phi_{1}>0 .
$$

To estimate $\Phi_{\mathbf{2}}$ we shall employ the method of complex integration. In (5) and (10) let us regard $z$ as a complex variable. This makes $[f(z)]^{1 / 2}$ and

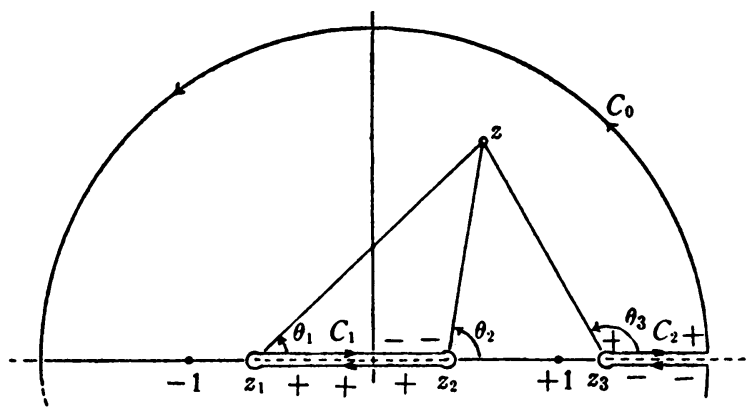

Diagram 2

$g_{2}(z)$ double-valued functions of $z$ which, however, are uniquely defined on the Riemann-sheet (diag. 2) bounded by the cuts $C_{1}$ and $C_{2}$ and the large circle $C_{0}$ with its centre at the origin $\left({ }^{8}\right)$. If we take on our sheet

$$
0 \leqq \arg \left(z-z_{k}\right)<2 \pi, \quad k=1,2,3,
$$

the sign of $[f(z)]^{1 / 2}$ on the cuts is as shown in the diagram.

We shall integrate $g_{2}(z)$ around the contours $C_{1}-C_{2}-C_{0}$. First we find that the complex integral

$$
\int_{C_{1}} g_{2}(z) d z=\int_{C_{1}}-\frac{\alpha}{2 A(z+1)[f(z)]^{1 / 2}} d z
$$

in which the sign of $[f(z)]^{1 / 2}$ is taken according to our convention (14), is equal to

$$
-2 \int_{z_{1}}^{z_{2}} g_{2}(z) d z=-2 \int_{z_{1}}^{z_{2}} \frac{\alpha}{2 A(z+1)[f(z)]^{1 / 2}} d z=-2 \Phi_{2}
$$

(8) These contours are due to Hadamard; see footnote 1. 
where $z$ is regarded as a real variable and the sign of $[f(z)]^{1 / 2}$ is taken as positive. tain

The residue of $g_{2}(z)$ at -1 is $-1 / 2 i$ so that by Cauchy's Theorem we ob-

$$
-2 \Phi_{2}+\int_{C_{2}} g_{2}(z) d z+\int_{C_{0}} g_{2}(z) d z=2 \pi i(-1 / 2 i)=-\pi .
$$

Now since $g_{2}(z)$ is real and positive on the upper side of $C_{2}$ and real and negative on the lower side (see diag. 2), $\int_{C_{2}}>0$; and $\int_{C_{0}} \rightarrow 0$ as the circle $C_{0}$ is made infinitely large. Therefore (15) yields $-2 \Phi_{2}<-\pi$ or

$$
\Phi_{2}>\pi / 2 \text {. }
$$

Adding (13) and (16) we arrive at Puiseux' inequality, namely

$$
\Phi=\Phi_{1}+\Phi_{2}>\pi / 2 \text {. }
$$

\section{I.2. An extension of Puiseux' inequality.}

TheOREM. When a spherical pendulum passes from its lower level, $z_{1}$, to the upper level, $z_{2}$, the advance in azimuth, $\Phi_{0}$, during that part of the motion which takes place in the lower hemisphere exceeds $\pi / 2$.

If $z_{2}$ happens to be in the lower hemisphere this theorem, of course, reduces to Puiseux' inequality. It covers every possible motion of the spherical pendulum since the well known inequality, $z_{1}+z_{2}<0\left(^{7}\right)$, implies that a part of the motion always takes place in the lower hemisphere. Let us also note an immediate consequence of our theorem and Halphen's inequality $(\Phi<\pi)\left({ }^{4}\right)$, namely that the advance in azimuth is greater in the lower hemisphere than in the upper one. When $z_{2}>0$ this means

$$
\phi(0)-\phi\left(z_{1}\right)>\phi\left(z_{2}\right)-\phi(0),
$$

in curious analogy to the inequality satisfied by the levels, which may be written in the form

$$
0-z_{1}>z_{2}-0 \text {. }
$$

Now for the proof. The case $z_{2} \leqq 0$ need not be discussed since it is covered by the proof of $\S I .1\left(^{9}\right)$. When $z_{2}>0$ we use the contours shown in diag. 3 . Applying contour-integration to $g(z)$, equation (6), we obtain

$$
-2 \Phi_{0}+\int_{L_{1}} g(z) d z+\int_{L_{2}} g(z) d z=-\pi .
$$

It is plain from this equation that $\int_{L_{1}}+\int_{L_{2}}$ is a real number. We wish to show that this number is positive. Let us call

( $\left.{ }^{9}\right)$ We could also use the vertical line of diag. 2; see footnote 6. 


$$
\begin{array}{llr}
\arg \left(z-z_{k}\right)=\theta_{k}, & k=1,2,3, \\
\arg g(z)=\omega, & \arg (z-1)=\epsilon_{1}, & \arg (z+1)=\epsilon_{2} .
\end{array}
$$

Equation (6) now permits us to express $\omega$ in terms of the other angles, namely

$$
\omega=\pi-\left(\epsilon_{1}+\epsilon_{2}+\left(\theta_{1}+\theta_{2}+\theta_{3}\right) / 2\right) .
$$

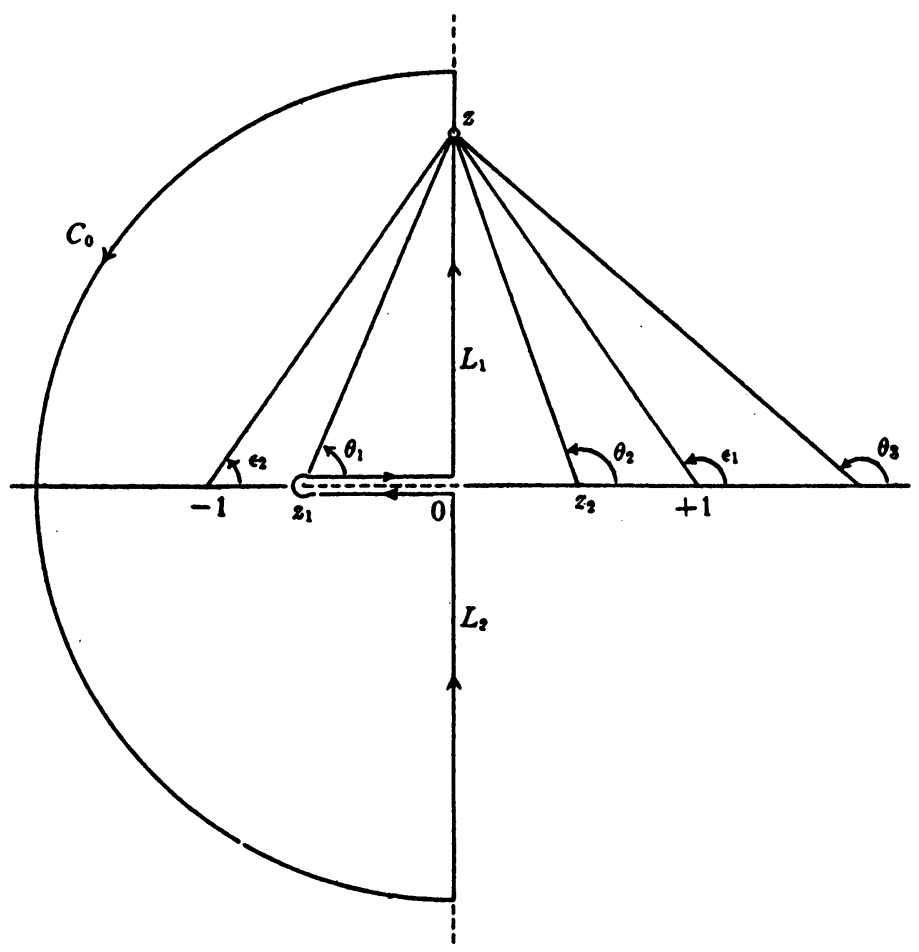

Diagram 3

If we remember that $z_{1}+z_{2}<0$ it is clear from diag. 3 that on $L_{1}$

$$
\epsilon_{1}+\epsilon_{2}=\pi, \quad \pi / 2<\theta_{1}+\theta_{2}<\pi, \quad \pi / 2<\theta_{3}<\pi .
$$

Substituting (21) into (20) we arrive at

$$
-\pi<\omega<-\pi / 2
$$

so that on $L_{1}$

$$
-\pi / 2<\arg [g(z) d z]<0 .
$$

It follows that the real part of $\int_{L_{1}}>0$; similarly we find that the real part of $\int_{L_{2}}>0$ so that the sum of these integrals is a real positive number as was to 
be shown. (18) now yields the desired result,

$$
\Phi_{0}>\pi / 2 \text {. }
$$

Such a result could not have been proven for the advance in azimuth up to a level below that of the equator. For if $\bar{z}$ is such a level, there will be motions whose $z_{1}=\bar{z}$, so that their advance in azimuth below $\bar{z}$ is 0 .

Another conceivable extension of our result would be that $\Phi_{0}$ always exceeded an angle which is greater than $\pi / 2$. But the fact that $\pi / 2$ has long been known to be the greatest lower bound even of $\Phi$ (cf. footnote 6), which is greater than $\Phi_{0}$, precludes this possibility.

Thus our result allows of no further generalization.

The increment of the angle of azimuth during the passage from any possible lower level $z_{1}$ to the fixed upper level $z_{2}$ has therefore a greatest lower bound $\mu\left(z_{2}\right)$ given by

$$
\begin{aligned}
\mu\left(z_{2}\right) & =0, & -1 \leqq z_{2}<0, \\
\mu\left(z_{2}\right) & =\pi / 2, & 0 \leqq z_{2} \leqq 1 .
\end{aligned}
$$

The findings of $\S \S I$ and I.2 and Halphen's well known result may be combined into the inequalities.

$$
\pi / 2<\Phi_{0} \leqq \Phi<\pi .
$$

Part II. The heavy symmetrical top

II.1. General. As in the case of the spherical pendulum we can decompose $g(z),(3)$, into two parts,

$$
g(z)=g_{1}(z)+g_{2}(z)
$$

where now

$$
g_{1}(z)=\frac{\beta-\alpha}{2 A} \frac{1}{(z-1)[f(z)]^{1 / 2}}
$$

and

$$
g_{2}(z)=\frac{\beta+\alpha}{2 A} \frac{1}{(z+1)\lfloor f(z)\rfloor^{1 / 2}}
$$

As before we call

$$
\int_{z 1}^{z 2} g_{k}(z)=\Phi_{k}, \quad k=1,2,
$$

so that

$$
\Phi=\Phi_{1}+\Phi_{2} .
$$

We are now in a position to apply contour-integration to $g(z), g_{1}(z)$ and $g_{2}(z)$ 
in order to derive bounds of $\Phi$ for the various types of motions of a heavy symmetrical top. For reasons which will presently beccome apparent these motions are best classified according to the value of the ratio $\alpha / \beta$. The sense of $\beta$ will be taken as positive throughout, so that, for example, $\Phi>0$ means that $\Phi$ has the same sense as $\beta$.

II.2. $\alpha / \beta<-1$. We integrate $g(z)$, (3), around the contour of diag. 2 and, making $C_{0}$ infinitely large, we obtain by Cauchy's theorem

$$
-2 \Phi+\int_{C_{2}} g(z) d z=2 \pi i \sum \text { residues. }
$$

As in $\$ I .1$ it is easily seen that the $\int_{C_{2}}$ is real and positive.

The residue of $g(z)$ at +1

$$
R(+1)=\lim _{z \rightarrow+1}(z-1) g(z)=-\frac{\alpha-\beta}{2 A[f(+1)]^{1 / 2}} .
$$

Now by (1), $[f(+1)]^{1 / 2}= \pm(\alpha-\beta) i / A$ and since $\arg [f(+1)]^{1 / 2}=\pi / 2$ (see (1) and (14)) we must choose the $-\operatorname{sign}\left({ }^{10}\right)$. Therefore

$$
R(+1)=1 / 2 i \text {. }
$$

Similarly

$$
R(-1)=1 / 2 i \text {. }
$$

Substituting (30) and (31) into (29) we obtain

$$
\Phi=-\pi+\frac{1}{2} \int_{C_{2}} g(z) d z>-\pi
$$

so that $-\pi$ is a lower bound of $\Phi$.

To determine an upper bound note that since during the entire motion from $z_{1}$ to $z_{2}, g_{1}(z)$. (considered as function of a real $z$ ) is negative, its integral

$$
\Phi_{1}=\int_{z 1}^{z 2} g_{1}(z) d z<0 .
$$

On the other hand, integrating $g_{2}(z)$ over the contours of diag. 2 we obtain

$$
\Phi_{2}=-\pi / 2+\int_{C_{2}} g_{2}(z) d z<-\pi / 2,
$$

which when combined with the inequality (33) gives the required upper bound

$$
\Phi=\Phi_{1}+\Phi_{2}<-\pi / 2 .
$$

As in the case of the spherical pendulum we can extend this inequality by

(10) The reason for classifying the motion according to the value of $\alpha / \beta$ is now plain. 
showing that even in the lower hemisphere the increment in azimuth

$$
\Phi_{0}<-\pi / 2 \text {. }
$$

The proof is not given here but it will be found identical with that of §II.4, except for the sign of the residue at -1 .

We therefore arrive at the following final result:

$$
-\pi<\Phi \leqq \Phi_{0}<-\pi / 2\left({ }^{11}\right) .
$$

II.3. $\alpha / \beta=-1$. In motions of this type the top passes through the lower position of rest, $z=-1$, as may be seen on setting $\alpha=-\beta$ in (1). This introduces a difficulty because the angle of azimuth, $\phi$, is not defined when $z= \pm 1$. We therefore can not longer maintain our original definition of $\Phi$,

$$
\Phi=\phi\left(z_{2}\right)-\phi\left(z_{1}\right) .
$$

Fortunately, however, our definition by means of an integral,

$$
\Phi=\int_{z 1}^{z_{2}} g(z) d z,
$$

is still good since in the present case $g(z)=g_{1}(z)$ and the latter is of the order of $(1+z)^{-1 / 2}$ near $z=-1$ (see (26)).

We may now apply contour-integration exactly as in $\S 1.2$ and thus arrive at the inequalities

$$
-\pi / 2<\Phi \leqq \Phi_{0}<0 .
$$

II.4. $-1<\alpha / \beta \leqq 0$. The lower bound of $\Phi$, in the present case, is easily seen to be 0 by integrating $g(z)$ over the contours of diag. 2(12). But the method' of decomposition which we might hope to give us the upper bound of $\Phi$ now merely yields the lower bound again and is therefore of no value. However, the method of $\S I .2$ will enable us to prove that $\Phi_{0}$ is less than $\pi / 2$; and noting that in the upper hemisphere $d \phi / d t$ is negative, we may then conclude that $\pi / 2$ is also an upper bound of $\Phi$.

Before proceeding to the proof proper we first establish two lemmas.

Lemma 1. The lower and upper levels, $z_{1}$ and $z_{2}$, of a heavy symmetrical top describing a motion in which $\alpha / \beta \leqq 0$, satisfy the inequality

$$
z_{1}+z_{2}<0 \text {. }
$$

The proof is simple: we pick out the coefficient of $z$ in $f(z),(1)$, and obtain

$$
z_{2} z_{3}+z_{3} z_{1}+z_{1} z_{2}=-1+\alpha \beta / A m g a
$$

or

(11) $\Phi \leqq \Phi_{0}$ follows from the constant negative sign of $d \phi / d t$, equation (2), when $\alpha<-\beta$.

(12) This was done by Hadamard; see footnote 1. 


$$
z_{1}+z_{2}=\left(-1-z_{1} z_{2}+\frac{\beta^{2}}{A m g a} \frac{\alpha}{\beta}\right) / z_{3}
$$

Observing now that $z_{3}>0, z_{1} z_{2}>-1\left({ }^{13}\right)$ and $\alpha / \beta \leqq 0$, we at once deduce our inequality (37).

LeMmA 2. Unless $z_{1}=\alpha / \beta= \pm 1$, the ratio $\alpha / \beta$ lies outside the interval $\left(-1, z_{1}\right)$.

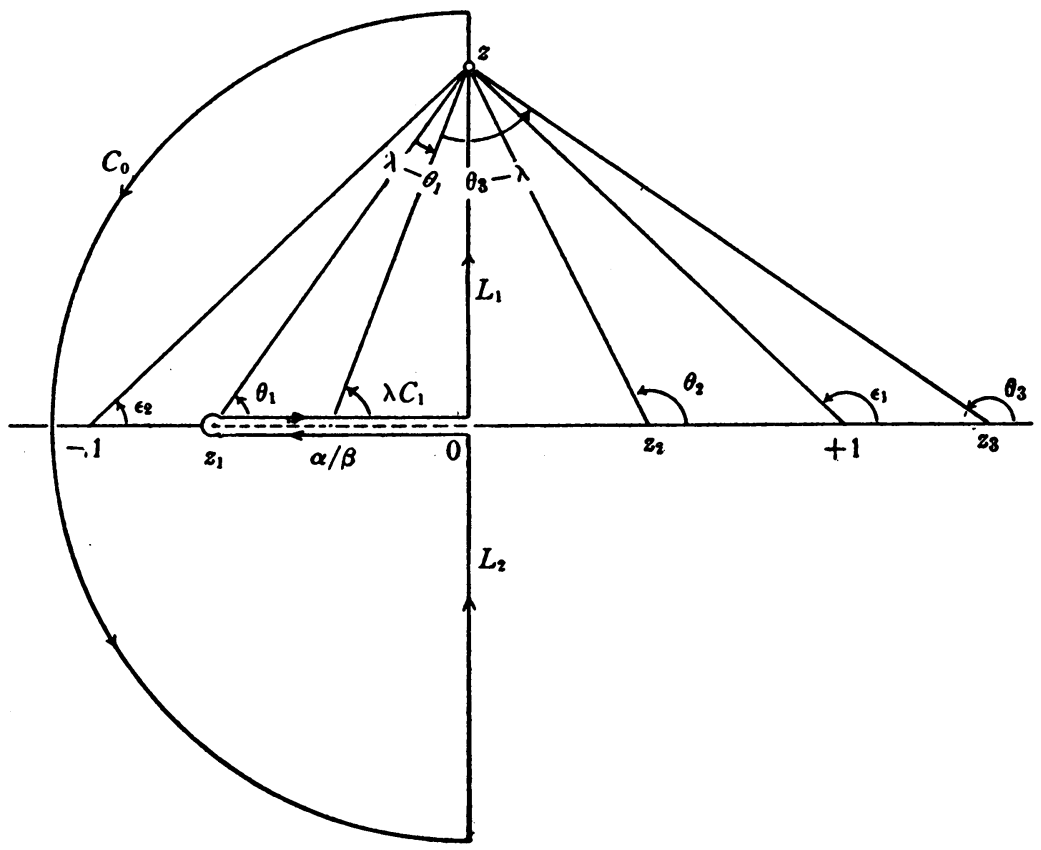

DIAGRAM 4

Let us suppose that our lemma was false and that a motion in which $-1<\alpha / \beta \leqq z_{1}$ could take place. Then integrating $g(z)$ around the contours of diag. 1 and observing that $R(+1)=1 / 2 i$ while $R(-1)=-1 / 2 i$ we obtain

$$
-2 \Phi+2 \beta \int_{z z}^{\infty} \frac{\alpha / \beta-z}{A\left(1-z^{2}\right)[f(z)]^{1 / 2}} \cdot d z=0, \text { or } \Phi>0 .
$$

But this is absurd because when $\alpha / \beta \leqq z_{1}, d \phi / d t \leqq 0$ during the entire motion (see (2)) so that $\Phi<0$. This demonstrates the truth of our lemma.

We are now prepared to return to our original question of finding an upper

(13) $z_{1} z_{2}=-1$ only when $z_{1}=-1$ and $z_{2}=+1$. In this case it follows from (1) that $\alpha=\beta=0$ so that $\alpha / \beta$ is indeterminate and not less than or equal to 0 as postulated. 
bound of $\Phi_{0}$. We first divide the motions under investigation into two categories according as $z_{2} \geqq 0$ and $z_{2}<0$. In motions of the first type we employ the contour shown in diag. 4. Integration of $g(z)$ over this contour gives (cf. (18))

$$
-2 \Phi_{0}+\int_{L_{1}} g(z) d z+\int_{L_{2}} g(z) d z=-\pi .
$$

It remains to be shown that $\int_{L_{1}}+\int_{L_{2}}<0\left({ }^{14}\right)$. With the notation of (19) and setting

$$
\arg (z-\alpha / \beta)=\lambda \text {, }
$$

the expression for $\omega$ derived from (4) is

$$
\omega=\lambda-\left(\epsilon_{1}+\epsilon_{2}+\left(\theta_{1}+\theta_{2}+\theta_{3}\right) / 2\right) .
$$

Since on $L_{1}$ the sum $\epsilon_{1}+\epsilon_{2}=\pi$ (see diag. 4), the argument, $\omega$, of $g(z)$ along that line may be written in either of the two following forms:

$$
\omega=\lambda-\left[\left(\theta_{1}+\theta_{2}\right)+\theta_{3}\right] / 2-\pi
$$

or

$$
\omega=-\theta_{2} / 2-\left[\left(\theta_{3}-\lambda\right)-\left(\lambda-\theta_{1}\right)\right] / 2-\pi .
$$

Now an inspection of diag. 4 shows that since by Lemma 1 (inequality (37)) $z_{1}+z_{2}<0$, the sum $\theta_{1}+\theta_{2}<\pi$. Also, it is clear that $\theta_{3}<\pi$ while $\lambda$ is, of course, positive. It therefore follows from the first expression for $\omega(40)$ that

$$
\omega>-2 \pi \text {. }
$$

On the other hand both $\theta_{2} / 2$ and the expression in brackets, (41), are positive (cf. diagram 4) so that from the second form of $\omega$ we have

$$
\omega<-\pi \text {. }
$$

(42) and (43) are combined in the inequality

$$
-2 \pi<\omega<-\pi \text {. }
$$

Noting now that, on the line $L_{1}$, arg $d z=\pi / 2$ we obtain from (44) that

$$
-3 \pi / 2<\arg [g(z) d z]<-\pi / 2 \text {. }
$$

Thus we see that on $L_{1}$ the real part of $g(z) d z$ is negative. Also, since $\omega(x-i y)$ $=5 \pi-\omega(x+i y)$ (cf. (39)), the same is true on $L_{2}$ so that what we set out to show is now plain, namely

$$
\int_{L_{1}} g(z) d z+\int_{L_{2}} g(z) d z<0
$$

(14) From (37) it follows, of course, that $\int_{L_{1}}+\int_{L_{2}}$ must be real. 
By substituting this inequality into (38) we obtain an upper bound of $\Phi_{0}$,

$$
\Phi_{0}<\pi / 2 \text {; }
$$

and remembering that $\Phi \leqq \Phi_{0}$ (cf. the first paragraph of $\left.\S I I .4\right)$ we also, automatically, have an upper bound for $\Phi$ :

$$
\Phi \leqq \Phi_{0}<\pi / 2 \text {. }
$$

We must still deal with motions in which $z_{2}<0$. The treatment is almost identical with the above, the only differences being that $C_{1}$ (diag. 4 ) is re-

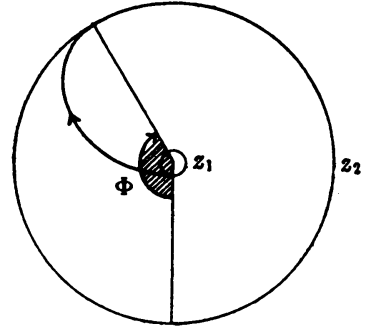

$\alpha / \beta=-1-0,-\pi<\Phi<-\pi / 2$

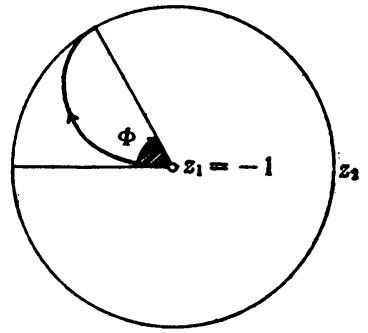

$\alpha / \beta=-1,-\pi / 2<\Phi<0$

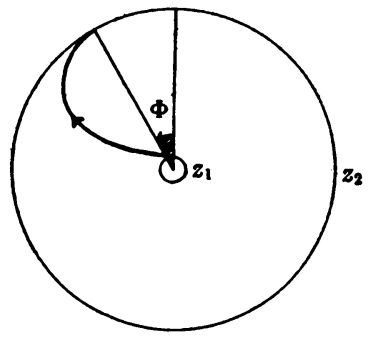

$\alpha / \beta=-1+0,0<\Phi<\pi / 2$

\section{Diagram 5}

placed by the complete Riemann-cut enclosing $z_{1}$ and $z_{2}$ and $L_{1}, L_{2}$ merge into a single line through the origin. The results are again the inequalities (45).

Thus we have in all motions of the type considered in this section $(-1<\alpha / \beta \leqq 0)$

$$
0<\Phi \leqq \Phi_{0}<\pi / 2 .
$$

If we look back at the results of the last three sections (inequalities (35), (36) and (46)) we notice that as the ratio $\alpha / \beta$ passes through the value -1 , the range of variation of $\Phi$ jumps twice by $\pi / 2$. The reason for this discontinuity is to be found in the fact that when $\alpha / \beta=-1$, the lower level, $z_{1}=-1$. Diag. 5, showing the projections of three neighbouring paths on the plane of the equator, explains the results.

II.5. $0<\alpha / \beta<1$. Lower bound. In the motions with which we have dealt up to now, contour-integration methods have always yielded both a lower and an upper bound of $\Phi$ (and $\Phi_{0}$ ). However when $\alpha / \beta$ exceeds 0 we find that contour-integration provides us only with a lower bound. This is by no means an accident. It will presently be shown that when $\alpha / \beta>0$ the angles $\Phi$ have no finite upper bound independent of $\alpha / \beta$, and we cannot expect straight contour-integration to lead us to that result. All we can derive from this method are equations of the form 


$$
\pm \Phi\left(\text { or } \pm \Phi_{0}\right)=n \pi / 2+\text { some integral, } \quad n=0,1 \text { or } 2,
$$

and without proving that the integrals have no upper bound it is impossible to show that $\Phi$ (or $\Phi_{0}$ ) is unbounded. But, if we do have to make an estimate of the upper bound of a set of integrals we might as well deal with the integrals (4) for $\Phi$ itself, which we shall actually do in $\$ I I .8$. In the present section and the two immediately following it only lower bounds will be derived.

For motions in which $0<\alpha / \beta<1$ we need merely quote Hadamard's result $\left({ }^{1}\right)$, which he derived by the same method as that sketched at the beginning of $\S I 1.4$. It is

$$
\Phi>0 \text {. }
$$

II.6. $\alpha / \beta=1$. Lower bound. This case deserves special interest because it covers the motions of a sleeping top, stable or unstable, when disturbed by an impulse.

When $\alpha / \beta=+1$ it follows from (1) that $z=+1$ is a root (possibly double) of $f(z)=0$. We have then the following subcases: Either both $z_{2}$ and $z_{3}$ are +1 , which gives the well known logarithmic (or aperiodic) motion of the top $\left({ }^{15}\right)$ with $\Phi=\infty$ (see $\left.\$ I I .8\right)$; or $z_{2}=1$ and $z_{3}>1$ in which case the top passes through the vertical position and as in $\S I I .3$ we must fall back on the integral definition (4) of $\Phi$, since $\phi\left(z_{2}\right)=\phi(+1)$ is meaningless; or, thirdly, we could have $z_{2}<+1$ and $z_{3}=+1$ in which case $\phi\left(z_{2}\right)$ is perfectly well defined and no further difficulty is introduced.

(25) shows that $g_{1}(z)=0$ so that $g(z)=g_{2}(z)$. Unless $z_{2}=z_{3}=+1$ we may now integrate over Hadamard's contours (diag. 2) $\left({ }^{16}\right)$ and thus obtain

whence

$$
-2 \Phi+\int_{C_{2}} g_{2}(z) d z=-\pi
$$

$$
\Phi>\pi / 2 \text {. }
$$

When $z_{2}=z_{3}=+1, \Phi=\infty\left({ }^{16}\right)$ (cf. (52)) so that the inequality (48) holds generally for all motions with $\alpha / \beta=+1$.

II.7. $\alpha / \beta>1$. Lower bound. Let us hrst try to integrate $g(z)$ over Hadamard's contours (diag. 2). This gives

or

$$
-2 \Phi+\frac{\beta}{A} \int_{C_{2}} \frac{\alpha / \beta-z}{\left(1-z^{2}\right)[f(z)]^{1 / 2}} d z=-2 \pi
$$

$$
\Phi=\pi+\frac{\beta}{A} \int_{z_{z}}^{\infty} \frac{\alpha / \beta-z}{\left(1-z^{2}\right)[f(z)]^{1 / 2}} d z
$$

(15) Klein and Sommerfeld, Ueber die Theorie des Kreisels, 1898, Teubner, pp. 334-336.

(16) When $z_{2}=z_{3}$, two distinct cuts, $C_{1}$ and $C_{2}$, are not possible. 
Now when $z_{3} \geqq \alpha / \beta$ the integrand in (49) is everywhere non-negative so that plainly

$$
\Phi>\pi \text {. }
$$

But when $z_{3}<\alpha / \beta$, the integrand changes sign at $z_{3}=\alpha / \beta$ and we cannot draw any immediate conclusions as to the value of the integral.

We therefore take recourse to our method of decomposition. Regardless of whether $z_{3}$ is smaller or greater than $\alpha / \beta$ this method leads to the following results:

$$
-2 \Phi_{1}<0
$$

and

$$
-2 \Phi_{2}=-\pi-\frac{\alpha+\beta}{A} \int_{z_{3}}^{\infty} \frac{1}{(z+1)[f(z)]^{1 / 2}} d z<-\pi .
$$

On addition we find

$$
\Phi=\Phi_{1}+\Phi_{2}>\pi / 2 .
$$

A check on this inequality can be obtained by setting $\beta=+0$. This reduces the top to a spherical pendulum for which the inequality (51) is known to hold true.

II.8. $0<\alpha / \beta<1 ; \alpha / \beta=1 ; \alpha / \beta>1$. Upper bounds. Our list of bounds of $\Phi$ is now complete, except for the upper bounds in motions in which $\alpha / \beta>0$. These form the topic of the present section. In (a) we shall show that, when $\alpha / \beta=1$, there are certain types of motion in which $\Phi=\infty$ and that, when $\alpha / \beta \rightarrow 1 \pm 0, \Phi$ can assume arbitrarily large values. In (b) the question of an upper bound of $\Phi$ for arbitrary, fixed values of $\alpha / \beta(0<\alpha / \beta<1$ and $1<\alpha / \beta$ $<\infty$ ) will be discussed.

(a) Beginning with the case $\alpha / \beta=+1$, we consider a particular motion of this kind, namely the aperiodic motion of the "weak" top. It is characterized by $z_{2}=z_{3}=+1$ and $z_{1}<+1$, and setting $(2 m g a A)^{1 / 2}=K$ (cf. (1)) the advance in azimuth, $\Phi$, is given by

(52) $\Phi=\int_{z 1}^{1} g(z) d z=\int_{z 1}^{1} g_{2}(z) d z=\frac{\beta}{K} \int_{z 1}^{1} \frac{1}{(1+z)\left(z-z_{1}\right)^{1 / 2}} \cdot \frac{d z}{1-z}=+\infty$.

Therefore no finite upper bound of $\Phi$ can be assigned in motions in which $\alpha / \beta=+1$.

This naturally leads us to suspect that if, in the motion of a weak top, we cause an infinitesimal change of the ratio $\alpha / \beta$, so that $\alpha / \beta=+1+0$ or $+1-0, \Phi$ will still be unbounded. In the case where $\alpha / \beta=+1+0$ this fact is easily verified. But when $\alpha / \beta=+1-0$ we find ourselves confronted with the necessity of making an estimate of the limit of an integral which we shall carry out by reference to the following lemma. 
LEMMA 3. If the three parameters $\alpha, \beta$ and $E$ represent a motion of $a$ heavy symmetrical top with cubic $f(z)$ (see (1)), then the parameters

$$
\alpha^{\prime}=\beta, \quad \beta^{\prime}=\alpha, \quad E^{\prime}=E+\frac{\alpha^{2}-\beta^{2}}{2}\left(\frac{1}{C}-\frac{1}{A}\right)
$$

determine a "conjugate" $\left.{ }^{17}\right)$ motion with the same cubic.

Since $E$ cannot be quite freely assigned, once $\alpha$ and $\beta$ are chosen, let us rather consider motions given by completely arbitrary initial conditions. Thus imagine that a motion is started at a level $z_{0}$, with angular momentum components $\alpha$ and $\beta$ and an initial vertical "velocity" $(d z / d t)_{0}$. The third parameter, $E$, can be expressed in terms of these quantities, by using (1):

$$
2 E=\frac{A}{1-z_{0}^{2}}\left(\frac{d z}{d t}\right)_{0}^{2}+\frac{\beta^{2}}{C}+\frac{\left(\alpha-\beta z_{0}\right)^{2}}{A\left(1-z_{0}^{2}\right)}+2 m g a z_{0} .
$$

Now take the same top and start it at the same initial level, $z_{0}$, as before, with the same $(d z / d t)_{0}$ but with new values of $\alpha$ and $\beta$, namely $\alpha^{\prime}=\beta$ and $\beta^{\prime}=\alpha$. The total energy, $E^{\prime}$, is now given by

$$
2 E^{\prime}=\frac{A}{1-z_{0}^{2}}\left(\frac{d z}{d t}\right)_{0}^{2}+\frac{\alpha^{2}}{C}+\frac{\left(\beta-\alpha z_{0}\right)^{2}}{A\left(1-z_{0}^{2}\right)}+2 m g a z_{0}
$$

and if we subtract (54) from (55) we obtain $2 E^{\prime}=2 E+\left(\alpha^{2}-\beta^{2}\right)(1 / C-1 / A)$ in agreement with (53). What is the significance of this result? It is, that if a motion with parameters $\alpha, \beta$ and $E$ is physically possible so is its "conjugate" motion with parameters $\alpha^{\prime}, \beta^{\prime}$ and $E^{\prime}$ as given by (53).

We must now merely convince ourselves that the new parameters, $\alpha^{\prime}, \beta^{\prime}$ and $E^{\prime}$, lead to the same cubic as $\alpha, \beta$ and $E$, which is easily done by substitution into (1).

Let us now return to our original problem and first investigate the case $\alpha / \beta=+1+0$. Let $\alpha, \beta(\alpha=\beta)$ and $(d z / d t)_{0}$ be the constants corresponding to the motion of a weak top. Of these three arbitrary parameters we leave $\beta$ and $(d z / d t)_{0}$ unchanged but increase $\alpha$ to $\alpha+\epsilon$, where $\epsilon$ is a small positive quantity, so that $(\alpha+\epsilon) / \beta>1$. Since now +1 is no longer a root of the new cubic $f(z)$ we must have $z_{2}=1-\gamma_{2}$ and $z_{3}=1+\gamma_{3}$, where $\gamma_{2}$ and $\gamma_{3}$ tend to 0 with $\epsilon$. The increment in azimuth in this motion is, by (4),

$$
\begin{aligned}
\Phi & =\frac{\beta}{K} \int_{z 1}^{z 2} \frac{(1+\epsilon / \beta-z)}{\left(1-z^{2}\right)\left(z-z_{1}\right)^{1 / 2}\left(z-z_{2}\right)^{1 / 2}\left(z_{3}-z\right)^{1 / 2}} d z \\
> & \frac{\beta}{K} \int_{z 1}^{z 2} \frac{1}{(1+z)\left(z-z_{1}\right)^{1 / 2}} \frac{1}{\left(z-z_{2}\right)^{1 / 2}\left(z_{3}-z\right)^{1 / 2}} d z .
\end{aligned}
$$

(17) The term "conjugate" was chosen because the relationship between the two motions is symmetric. 
If we let $\epsilon \rightarrow 0$, the last integral approaches that of (52) which is infinite and thus we find that $\Phi \rightarrow \infty$ as $\alpha / \beta \rightarrow+1+0$.

The other case, where the new value of $\alpha$ is $\alpha-\epsilon$, is somewhat tricky. For now

$$
\begin{aligned}
\Phi= & \frac{\beta}{K} \int_{z 1}^{z 2} \frac{(1-\epsilon / \beta-z)}{\left(1-z^{2}\right)\left(z-z_{1}\right)^{1 / 2}\left(z-z_{2}\right)^{1 / 2}\left(z_{3}-z\right)^{1 / 2}} d z \\
= & \frac{\beta}{K} \int_{z 1}^{z 2} \frac{1}{(1+z)\left(z-z_{1}\right)^{1 / 2}} \frac{1}{\left(z-z_{2}\right)^{1 / 2}\left(z_{3}-z\right)^{1 / 2}} d z \\
& -\frac{\epsilon}{K} \int_{z 1}^{z_{2}} \frac{1}{(1+z)\left(z-z_{1}\right)^{1 / 2}} \frac{1}{(1-z)\left(z-z_{2}\right)^{1 / 2}\left(z_{3}-z\right)^{1 / 2}} d z .
\end{aligned}
$$

As $\epsilon \rightarrow 0$ the first term on the right-hand side of (56) clearly tends to the expression for $\Phi$ in (52) which is infinite. The limiting value of the second term however cannot be immediately determined since the integral tends to infinity while $\epsilon / K$ approaches 0 . Thus an estimatè of the limit of $\Phi$ from (56) would involve a rather delicate investigation into the dependence of $z_{2}$ and $z_{3}$ on $\epsilon$. Instead we shall employ Lemma 3 in the following indirect approach.

Let $\alpha, \beta$ and $E$ be the parameters of a motion in which $\alpha / \beta>1$; and $\alpha^{\prime}, \beta^{\prime}$, $E^{\prime}$ be the corresponding parameters of the conjugate motion. The respective increments in azimuth are given by

and

$$
\Phi=\int_{z 1}^{z_{2}} \frac{\alpha-\beta z}{A\left(1-z^{2}\right)[f(z)]^{1 / 2}} d z
$$

$$
\Phi^{\prime}=\int_{z 1}^{z_{2}} \frac{\alpha^{\prime}-\beta^{\prime} z}{A\left(1-z^{2}\right)[f(z)]^{1 / 2}} d z=\int_{z 1}^{z_{2}} \frac{\beta-\alpha z}{A\left(1-z^{2}\right)[f(z)]^{1 / 2}} d z .
$$

The difference between these angles is

$$
\delta=\Phi-\Phi^{\prime}=\int_{z 1}^{z 2} \frac{\alpha-\beta}{A(1-z)[f(z)]^{1 / 2}} d z .
$$

It can be estimated by integration over Hadamard's contours (diag. 1) which results in

$$
-2 \delta+2 \int_{z_{3}}^{\infty} \frac{\alpha-\beta}{A(1-z)[f(z)]^{1 / 2}} d z=-2 \pi
$$

Clearly $\int_{z_{3}}^{\infty}<0$ and therefore it follows that

$$
\delta=\Phi-\Phi^{\prime}<\pi .
$$

But we have proved above that $\Phi \rightarrow+\infty$ as $\alpha / \beta \rightarrow+1+0$ and the inequality 
(57) now shows that the same must be true for the conjugate motions in which $\alpha^{\prime} / \beta^{\prime} \rightarrow+1-0$.

(b) We shall now consider $\Phi$ for arbitrary values of $\alpha / \beta$ on the interval $0<\alpha / \beta<1$. Denoting the least upper bound of $\Phi$ corresponding to a given $\alpha / \beta$ by $U(\alpha / \beta)$ we shall show that when $0<\alpha / \beta<1, U(\alpha / \beta) \geqq \pi / 2$.

With the notation of $\$ I I .1$ we have

$$
\Phi_{1}=\frac{\beta}{2 A} \int_{z 1}^{z_{2}} \frac{1-\alpha / \beta}{(z-1)[f(z)]^{1 / 2}} d z ; \quad \Phi_{2}=\frac{\beta}{2 A} \int_{z 1}^{z 2} \frac{1+\alpha / \beta}{(z+1)[f(z)]^{1 / 2}} d z \text {. }
$$

By contour-integration over Hadamard's contours (diag. 2) we find

$$
\Phi_{2}=\pi / 2+\frac{\beta}{2 A} \int_{z_{z}}^{\infty} \frac{1+\alpha / \beta}{(z+1)[f(z)]^{1 / 2}} d z .
$$

If we set $\Phi-\pi / 2=\eta$, we obtain on division by $\beta$

$$
\begin{aligned}
\eta / \beta=\left(\Phi_{1}+\Phi_{2}-\pi / 2\right) / \beta= & \frac{1}{2 A} \int_{z_{1}}^{z_{2}} \frac{1-\alpha / \beta}{(z-1)[f(z)]^{1 / 2}} d z \\
& +\frac{1}{2 A} \int_{z_{z}}^{\infty} \frac{1+\alpha / \beta}{(z+1)[f(z)]^{1 / 2}} d z .
\end{aligned}
$$

We aim to establish the existence of a sequence of motions in which $\alpha / \beta=$ const. and, as $\beta \rightarrow 0$, the right-hand side of (58) approaches a finite limit. It would then follow that in this sequence $\lim _{\beta \rightarrow 0} \eta=0$ and $\lim \Phi=\pi / 2$, which in turn would prove that $U(\alpha / \beta) \geqq \pi / 2$.

Consider the sequence of motions defined as follows:

$$
\begin{aligned}
\alpha / \beta & =\text { an arbitrary const. in the interval } 0<\alpha / \beta<1, \\
z_{0} & =\text { any const. in the interval }-1<z_{0}<1, \\
(d z / d t)_{0} & =0, \\
\beta_{n} & \rightarrow 0 .
\end{aligned}
$$

As $n \rightarrow \infty, \alpha$ and $\beta$ both tend to 0 , so that the cubic $f(z)$, equation (1), approaches the limiting cubic

$$
\bar{f}(z)=(1 / A)(2 E-2 m g a z)\left(1-z^{2}\right),
$$

whose roots are $-1,+1$, and $E / m g a$. To find the value of $E / m g a$ set $z=z_{0}$ in (60), resulting in

$$
0=(1 / A)\left(2 E-2 m g a z_{0}\right)\left(1-z_{0}^{2}\right)
$$

whence

$$
E / m g a=z_{0} .
$$

Thus the roots of the limiting cubic, $\bar{f}(z)$, are $\bar{z}_{1}=-1, \bar{z}_{2}=z_{0}, \bar{z}_{3}=+1$. A late 
member $f_{n}(z)$ in the sequence (59) will be of the type shown in diag. 6(a). Setting $K=+(2 m g a A)^{1 / 2}$, we have from (58):

$$
\begin{aligned}
\lim _{\beta \rightarrow 0} \eta / \beta= & \frac{1}{2 K} \int_{-1}^{z_{0}} \frac{1-\alpha / \beta}{(z-1)\left[(z+1)\left(z-z_{0}\right)(z-1)\right]^{1 / 2}} d z \\
& +\frac{1}{2 K} \int_{1}^{\infty} \frac{1+\alpha / \beta}{(z+1)\left[(z+1)\left(z-z_{0}\right)(z-1)\right]^{1 / 2}} d z .
\end{aligned}
$$

The integrand in the $\int_{-1}^{z_{0}}$ is finite at all points of the range of integration except

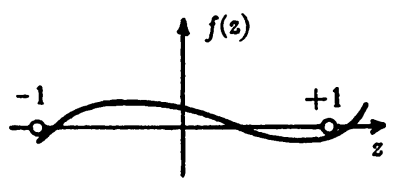

(a)

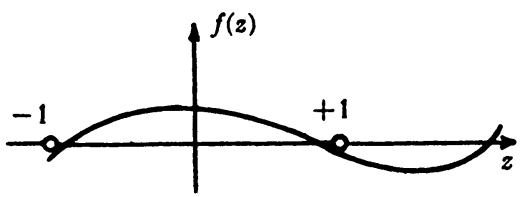

(b)

\section{Diagram 6}

at -1 and $z_{0}$, where it is of the order $(z-a)^{-1 / 2}$. Therefore $\int_{-1}^{z_{0}}$ is finite. Similarly $\int_{1}^{\infty}$ is finite. Therefore

$$
\lim _{\beta \rightarrow 0} \eta=0 ; \quad \lim \Phi=\pi / 2, .
$$

so that $U(\alpha / \beta) \geqq \pi / 2$. Also from its definition as least upper bound $U(\alpha / \beta)$ must be single-valued. Combining our results with earlier findings (cf. $\S I I .4$, II.8) we may write:

$$
U(+0)=\pi / 2, \quad U(\alpha / \beta) \geqq \pi / 2, \quad 0<\alpha / \beta<1, \quad U(1-0)=+\infty .
$$

This shows that in contrast to the motions with $\alpha / \beta<-1$ or $-1<\alpha / \beta \leqq 0$, in which a finite upper bound existed independent of the particular value of $\alpha / \beta, U(\alpha / \beta)$ for $0<\alpha / \beta<1$ is a nonconstant function of $\alpha / \beta$ (cf. diag. 1). An expression for $U(\alpha / \beta)$ has not been found.

Turning now to the case of motions with $1<\alpha / \beta$ we have (49), and setting $\Phi-\pi=\kappa$,

$$
\kappa / \beta=\frac{1}{A} \int_{z_{3}}^{\infty} \frac{\alpha / \beta-z}{\left(1-z^{2}\right)[f(z)]^{1 / 2}} d z .
$$

Consider the sequence of motions given by

$$
\begin{aligned}
\alpha / \beta & =\text { an arbitrary const. }>1, \\
z_{0} & =0, \\
(d z / d t)_{0} & =+(2 m g a c / A)^{1 / 2}, \quad c>1, \\
\beta_{n} & \rightarrow 0 .
\end{aligned}
$$


The limiting cubic is again given by $(60)$ and has the roots $-1,+1$, and $E / m g a$. To find the value of the latter set $z=z_{0}=0$ in (60). This results in

$$
2 m g a c / A=2 E / A \text {, }
$$

whence $E / m g a=c>1$. Therefore the roots of the limiting cubic, $\bar{f}(z)$, are now $\bar{z}_{1}=-1, \bar{z}_{2}=+1, \bar{z}_{3}=c>1$, and a late member $f_{n}(z)$ of the sequence (61) is of the type shown in diag. $6(\mathrm{~b})$. For $\lim _{\beta \rightarrow 0} \kappa / \beta$ we find:

$$
\lim _{\beta \rightarrow 0} \kappa / \beta=\frac{1}{K} \int_{c}^{\infty} \frac{\alpha / \beta-z}{\left(1-z^{2}\right)[(z+1)(1-z)(z-c)]^{1 / 2}} d z .
$$

Since the integrand in (62) is finite over the range of integration except at $z=c$, where it is of the order $(z-c)^{-1 / 2}$, and since it is of the order of $z^{-5 / 2}$ for $z=\infty, \int_{c}^{\infty}$ is finite. Therefore

$$
\lim _{\beta \rightarrow 0} \kappa=0 ; \quad \lim _{\beta \rightarrow 0} \Phi=\pi,
$$

so that for $\alpha / \beta>1, U(\alpha / \beta) \geqq \pi$. Again $U(\alpha / \beta)$ must needs be single-valued. Furthermore, since $\alpha / \beta=\infty$ leads us to the motion of a spherical pendulum we know by $(23)$ that $U(\infty)=\pi$. Finally, by $\S I I .8, U(1+0)=\infty$. Collecting these results we have

$$
U(1+0)=+\infty, \quad U(\alpha / \beta) \geqq \pi, \quad 1<\alpha / \beta<\infty, \quad U(\infty)=\pi
$$

(see diag. 1). An expression for $U(\alpha / \beta)$ on the interval $1<\alpha / \beta<\infty$ is not known.

II.9. Exact bounds. It will be shown in this section that the bounds of $\Phi$ established in $\$ \S I I .2$ to II.8 are actually the greatest lower and least upper bounds respectively.

Let us consider, as a typical example, any motion with a ratio $\alpha / \beta<-1$. By (32) we find for the excess of $\Phi$ over $-\pi$, which we shall denote by $\nu$,

$$
\nu=\frac{1}{2} \int_{C_{2}} g(z) d z=\frac{1}{A} \int_{z_{3}}^{\infty} \frac{\alpha-\beta z}{\left(1-z^{2}\right)[f(z)]^{1 / 2}} d z
$$

and dividing through by $\beta$ we obtain

$$
\nu / \beta=\frac{1}{A} \int_{z_{z}}^{\infty} \frac{\alpha / \beta-z}{\left(1-z^{2}\right)[f(z)]^{1 / 2}} d z .
$$

We now consider the sequence of motions

$$
\begin{aligned}
\alpha / \beta & =\text { const. }<-1, \\
z_{0} & =0, \\
(d z / d t)_{0} & =+(2 m g a c / A)^{1 / 2}, \quad c>1, \\
\beta_{n} & \rightarrow 0,
\end{aligned}
$$


and exactly as in $\S I I .8(b)$ in the discussion of $\kappa$ we can show that $\lim _{\beta \rightarrow 0} \nu=0$; $\lim _{\beta \rightarrow 0} \Phi=-\pi$ so that $-\pi$ is actually the greatest lower bound of all $\Phi$ 's corresponding to: any given value $\alpha / \beta<-1$.

A late member of the sequence of motions (63), whose $\Phi \sim-\pi$, has a cubic of the type sketched in diag. $6(\mathrm{~b})\left({ }^{18}\right)$.

Similarly, using (33) and (34), we find for the difference, $\rho$, between $-\dot{\pi} / 2$ and $\Phi$

$$
\rho=\frac{1}{2 A} \int_{z 1}^{z z} \frac{\beta-\alpha}{(z-1)[f(z)]^{1 / 2}} d z-\frac{1}{2 A} \int_{z_{3}}^{\infty} \frac{\beta+\alpha}{(z+1)[f(z)]^{1 / 2}} d z
$$

and we can easily verify that in the sequence of motions defined by

$$
\begin{array}{rlrl}
\alpha / \beta & =\text { const. }<-1, & \\
z_{0} & =0, & & \\
(d z / d t)_{0} & =0, & & \\
\beta_{n} & \rightarrow 0, & & \\
\lim _{\beta \rightarrow 0} \rho & =0 ; & \quad \lim _{\beta \rightarrow 0} \Phi=-\pi / 2
\end{array}
$$

(cf. $\S I$ I.8(b), discussion of $\eta$ ). Thus $-\pi / 2$ is the least upper bound of all $\Phi$ 's corresponding to a given $\alpha / \beta<-1$.

A late member of the sequence of motions (64), whose $\Phi \sim-\pi / 2$, has a cubic of the type shown in diag 6(a) $\left({ }^{19}\right)$.

In all other motions our contention, that the bounds of $\Phi$ established in $\$ \S I I .2$ to II. 8 are exact, may be verified by analogous methods. Where $L(\alpha / \beta)$ and $U(\alpha / \beta)$ are exactly known, motions, in which $\Phi$ approaches these bounds to an arbitrary degree of approximation, are listed in Table III.

TABLE III

Motions in which $\Phi \sim L(\alpha / \beta), U(\alpha / \beta)$.

\begin{tabular}{|c||c|c|c|c|c|c|c|c|c|}
\hline$\alpha / \beta$ & \multicolumn{2}{|c|}{$\alpha / \beta<-1$} & \multicolumn{2}{|c|}{$\alpha / \beta=-1$} & $-1<\alpha / \beta \leqq 0$ & $0<\alpha / \beta<1$ & $\alpha / \beta=1$ & $\alpha / \beta>1$ \\
\hline$z_{0}$ & 0 & 0 & 0 & 0 & 0 & 0 & 0 & 0 & 0 \\
\hline$(d z / d i)_{0}$ & $V$ & 0 & $V$ & 0 & $V$ & 0 & $V$ & $V$ & 0 \\
\hline$\beta$ & +0 & +0 & +0 & +0 & +0 & +0 & +0 & +0 & +0 \\
\hline \hline$\Phi$
\end{tabular}

(18) The angle $\Phi$ is made up of increments $\sim-\pi / 2$ near each pole, since $d \phi / d z$, equation (3), tends to 0 at all intermediate points.

(19) The angle $\Phi$ is described almost exclusively in the neighbourhood of $z=-1$, contributions of other parts of the path tending to 0 with $\beta$. 
II.10. Continuity of $\Phi$ for a fixed $\alpha / \beta$. For completeness sake we shall now give a formal proof of the physically plausible fact that, for a given $\alpha / \beta$, $\Phi$ takes all values between its greatest lower bound $L(\alpha / \beta)$ and least upper bound $U(\alpha / \beta)$. Let us first again consider a motion with $\alpha / \beta<-1$. We pick a late member, corresponding to $n=N$, from each of the sequences (63) and (64). Let us denote the motion taken from the sequence (64) by $M(0)$, and that taken from (63) by $M(1)$. Then we know from $\S I I .9$ that in $M(0)$ $\Phi=\Phi(0)=-\pi / 2-\delta_{0}$, and in $M(1), \Phi=\Phi(1)=-\pi+\delta_{1}$, where $\delta_{0}, \delta_{1}>0$ and $\delta_{0}, \delta_{1} \rightarrow 0$ as $N \rightarrow \infty$. But $M(0)$ and $M(1)$ may be regarded as extreme elements of a set of motions defined by

$$
M(s) \equiv\left[\begin{array}{rlrl}
\alpha / \beta & =\text { const. }<-1, & & z_{0}=0, \\
(d z / d t)_{0}=s(2 m g a c / A)^{1 / 2} & (c>1, \quad 0 \leqq s \leqq 1), & \beta & =\beta_{N} .
\end{array}\right.
$$

Now since $\alpha / \beta \neq \pm 1, z_{1}$ and $z_{2} \neq \pm 1$ for all values of $s$. Therefore

$$
\Phi(s)=\int_{z 1}^{22} g(z ; s) d z
$$

is a continuous function of $s$ and assumes all values between $\Phi(0)=-\pi / 2-\delta_{0}$ and $\Phi(1)=-\pi+\delta_{1}$. As $N \rightarrow \infty, \delta_{0}, \delta_{1} \rightarrow 0$ so that $\Phi(s ; N)$ assumes all values $\Phi$ satisfying the inequality $-\pi<\Phi<-\pi / 2$.

The same type of argument may be applied to any $\alpha / \beta$ giving rise to finite values of $\Phi$ only, that is, any $\alpha / \beta \neq 1$. In all these cases it may be verified that $\Phi$ assumes every value between its lower and upper bound.

It remains to investigate motions with $\alpha / \beta=1$, in which, as we have seen in $\S I I .8, \Phi$ may be infinite. Table III shows that when

$$
\begin{aligned}
\alpha / \beta & =1, & z_{0} & =0, \\
(d z / d t)_{0} & =(2 m g a c / A)^{1 / 2}, \quad c>1, & \beta & =\beta_{N},
\end{aligned}
$$

$\Phi=\pi / 2+\delta$, where $\delta$ is a positive number tending to zero as $\beta_{N} \rightarrow 0$. This motion has a cubic of the type of diag. $6(\mathrm{~b})$ with roots

$$
z_{1}=-1+0 ; \quad z_{2}=1 ; \quad z_{3} \sim c
$$

(cf. (60)).

On the other hand the motion given by

$$
\begin{aligned}
\alpha / \beta & =1, & z_{0} & =0, \\
(d z / d t)_{0} & =0, & \beta & =\beta_{N}
\end{aligned}
$$

has a cubic of the general shape shown in diag. 6(a) with roots

(cf. (60)).

$$
z_{1}=-1+0 ; \quad z_{2} \sim 0 ; \quad z_{3}=1
$$


It is therefore clear that in the one-parameter set of motions given by

$$
M(s) \equiv\left[\begin{array}{rlrl}
\alpha / \beta & =1, & z_{0}=0, \\
(d z / d t)_{0}=+s(2 m g a c / A)^{1 / 2} & (c>1, \quad 0 \leqq s \leqq 1), & \beta & =\beta_{N}
\end{array}\right.
$$

there must be one, corresponding to $s=s_{0}$, say, which has a cubic with roots

$$
z_{1}=-1+0 ; \quad z_{2}=1 ; \quad z_{3}=1 .
$$

But these roots give rise to an aperiodic motion (cf. (52)) $\left({ }^{15}\right)$ with $\Phi=\infty$. Let us for brevity set $s_{0}(2 m g a c / A)^{1 / 2}=k$. Then since $\alpha / \beta=1$ implies $g_{1}(z)=0$ (cf. (25)) we have in the range $k<(d z / d t)_{0} \leqq(2 m g a c / A)^{1 / 2}$

$$
\Phi=\int_{z 1}^{1} g_{2}(z) d z=\frac{\beta_{N}}{K} \int_{z_{1}}^{1} \frac{1}{(z+1)\left[\left(z-z_{1}\right)(1-z)\left(z_{3}-z\right)\right]^{1 / 2}} d z
$$

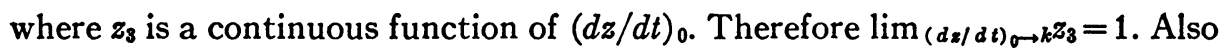
$\Phi$ is a continuous function of $z_{3}$ on the range considered, since $z_{3} \neq 1$ as long as $(d z / d t)_{0}>k$.

In order to prove that as $(d z / d t)_{0}$ varies from $(2 m g a c / A)^{1 / 2}$ to $k, \Phi$ assumes all values between $\pi / 2+\delta$ and $+\infty$, we must show that $\lim _{(d z / d t)_{0 \rightarrow k}} \Phi$ $=\infty$. We have:

Therefore:

$$
\begin{array}{rlrl}
\Phi & >\frac{\beta_{N}}{K} \int_{z 1}^{1} \frac{d z}{2^{3 / 2}(1-z)^{1 / 2}\left(z_{3}-z\right)^{1 / 2}} \\
& >\frac{\beta_{N}}{2^{3 / 2} \cdot K} \int_{0}^{1-\epsilon} \frac{d z}{(1-z)^{1 / 2}\left(z_{3}-z\right)^{1 / 2}}, & \epsilon>0 .
\end{array}
$$

$$
\begin{aligned}
\lim _{(d z / d t)_{0 \rightarrow K}} \Phi & >\frac{\beta_{N}}{2^{3 / 2} \cdot K} \cdot \lim _{(d z / d t)_{\bullet \rightarrow k}} \int_{0}^{1-\epsilon} \frac{d z}{(1-z)^{1 / 2}\left(z_{3}-z\right)^{1 / 2}} \\
& =\frac{\beta_{N}}{2^{3 / 2} \cdot K} \int_{0}^{1-\epsilon} \lim _{(d z / d t)_{\rightarrow} \rightarrow k} \frac{d z}{(1-z)^{1 / 2}\left(z_{3}-z\right)^{1 / 2}} \\
& =\frac{\beta_{N}}{2^{3 / 2} \cdot K} \int_{0}^{1-\epsilon} \frac{d z}{(1-z)}=\frac{\beta_{N}}{2^{3 / 2} \cdot K} \cdot[-\log \epsilon],
\end{aligned}
$$

for any small positive $\epsilon$. But the last expression is unbounded as $\epsilon \rightarrow 0$, so that $\lim _{(d z / d t)_{0 \rightarrow k} \Phi=\infty}$ as was to be proved. For sufficiently large values of $N$, $\delta$ becomes arbitrarily small, whence we conclude that when $\alpha / \beta=1, \Phi$ assumes all values satisfying the inequality $\Phi>\pi / 2$.

APPENDIX. The RETROgRAdELY PRECESSING heAvy SYMMETRICAL TOP AND THE SPHERICAL PENDULUM

In Parts I and II we have dealt independently first with the spherical pendulum and subsequently with the heavy symmetrical top. This scheme 
was adopted so that the reader who is not prepared to delve into the somewhat involved theory of tops can gather the principles of our methods from the simpler case of the spherical pendulum. But for this reason Part I would be completely superfluous, since a spherical pendulum may be regarded as a special case of a retrogradely precessing top $(\alpha / \beta<-1, \Phi$ negative; see $\S I I .2)$. We have shown for such a top that $-\pi<\Phi \leqq \Phi_{0}<-\pi / 2$, where the sense of $\alpha$ was regarded as negative. The same result must hold equally well for the spherical pendulum and if we now take $\alpha$ as positive it becomes the familiar $\pi / 2<\Phi_{0}<\Phi<\pi$, proved independently in Part I. Moreover $\pi / 2$ and $\pi$ must be the exact bounds of $\Phi$ (cf. §II.9).

Indeed we may observe that all important characteristics of the motion of the spherical pendulum, namely:

1. A unidirectional precession;

2. The level-inequality, $z_{1}+z_{2}<0$;

3. The extended Puiseux' inequality, $\Phi_{0}>\pi / 2$ (or $\Phi_{0}<-\pi / 2$, according to our choice of sign);

4. Halphen's inequality, $\Phi<\pi$ (or $\Phi>-\pi$ )

are shared by the retrogradely precessing top.

UNIVERSITY OF TORONTO,

Toronto, Ontario, Canada. 